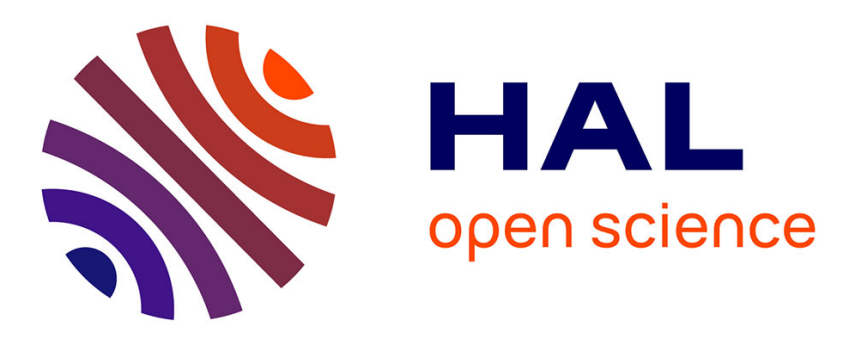

\title{
Addressing Organic Electrochemical Transistors for Neurosensing and Neuromorphic Sensing
}

\author{
Mahdi Ghazal, Thomas Dargent, Sébastien Pecqueur, F. Alibart
}

\section{To cite this version:}

Mahdi Ghazal, Thomas Dargent, Sébastien Pecqueur, F. Alibart. Addressing Organic Electrochemical Transistors for Neurosensing and Neuromorphic Sensing. IEEE SENSORS 2019, Oct 2019, Montréal, Canada. 10.1109/SENSORS43011.2019.8956648 . hal-03281012

\section{HAL Id: hal-03281012 https://hal.science/hal-03281012}

Submitted on 7 Jul 2021

HAL is a multi-disciplinary open access archive for the deposit and dissemination of scientific research documents, whether they are published or not. The documents may come from teaching and research institutions in France or abroad, or from public or private research centers.
L'archive ouverte pluridisciplinaire HAL, est destinée au dépôt et à la diffusion de documents scientifiques de niveau recherche, publiés ou non, émanant des établissements d'enseignement et de recherche français ou étrangers, des laboratoires publics ou privés. 


\section{Addressing Organic Electrochemical Transistors for Neurosensing and Neuromorphic Sensing}

\author{
Mahdi Ghazal \\ École Supérieure \\ d'Ingénieurs en \\ Électrotechnique et \\ Électronique, Paris, France \\ Institut d'Électronique, \\ Microélectronique et \\ Nanotechnologie, \\ Villeneuve d'Ascq, France
}

\author{
Thomas Dargent \\ Institut d'Électronique, \\ Microélectronique et \\ Nanotechnologie, \\ Villeneuve d'Ascq, France
}

\author{
Sebastien Pecqueur* \\ Institut d'Électronique, \\ Microélectronique et \\ Nanotechnologie, \\ Villeneuve d'Ascq, France \\ sebastien.pecqueur@iemn.u \\ niv-lille1.fr
}

\author{
Fabien Alibart \\ Institut d'Électronique, \\ Microélectronique et \\ Nanotechnologie, \\ Villeneuve d'Ascq, France \\ University of Sherbrooke, \\ QC, Canada
}

\begin{abstract}
We report on the comparison of two different driving circuits for addressing micro-fabricated organic electrochemical transistors of different channel resistances and transconductance, aiming for neuromorphic sensing. While the Current-Voltage converter observed faster transients, the Wheatstone bridge configuration offers more versatility towards higher resistance materials. Both circuits shows different assets very encouraging for further practical application.
\end{abstract}

Keywords-OECT; sensing circuit; signal read-out; currentvoltage converter; Wheatstone bridge

\section{INTRODUCTION}

In the quest of monitoring living matter activities, recording of extracellular potentials in electroactive cells represents a major challenge. For instance, this sensing approach can open various application fields, from health diagnosisfor (e.g. brain disease) to bioelectronics communication (i.e. Brain Machine Interfaces). Lately, the performance of organic bioelectronics, particularly organic electrochemical transistors (OECTs), has received lots of attention for extracellular recordings since they exhibit a better coupling with the cells, improve biocompatibility and better signal-to-noise ratio since they can be considered as active elements.[1] In addition, OECTs have already been used in various biomolecular sensors applications to measure glucose,[2] dopamine,[3] lactate,[4] and bacteria.[5] Nevertheless, OECT's applications for monitoring bioelectrochemical activity in vivo or in vitro [6] or to implement ionic sensor arrays are still at an early stage and integrating such devices into large systems required addtionnal research efforts.

The operation mechanism of a PEDOT:PSS OECT is primarily based on the electrochemical doping/de-doping processes when ions are injected/removed from the active material by applying an electric field on the gate terminal. Ions modulate the electrical conductivity of the semiconductor organic material by oxidation/reduction.[7] The resulting doping level can then be monitored by applying a source-drain voltage bias to promote a current drift across the channel.

For instance, when recording electrical activity from.in invitro neural cells cultures,[8] electroactive cells can be considered as a power supply in the ionic circuit that produce transient voltages that will be translated into modulation in the output drain current. Also, measuring the transient response of OECTs to pulse of voltages have been demonstrated as a promising route for neuromorphic sensing and neuromorphic computing giving access to a larger set of information and Hence, OECTs as a sensor produces a change in its electrical conductivity to indicate a change in its chemical or biological environment. To record this change in electrical property, the current output signal needs to be conditioned by an analog circuit. Such additional sensing circuitry required further analysis since it will be an important element of the overall measurement system in any applications. Notably, such additional circuitry need to be adapted to OECTs specificity such as dynamic range of operation and variability among devices. In this paper, we explore different analog circuits for sensing electrical signals from OECT devices.

\section{OECT ELECTRICAL CHARACTERISTICS}

In the output curves of the OECTs (displayed in Fig. 1(a)), the drain current increases linearly with the drain voltage, maintaining an Ohmic behavior from 0 to $1 \mathrm{~V}$. The characterized devices have shown different channel resistance values from $100 \Omega$ to $10 \mathrm{G} \Omega$ as shown in the histogram in Fig. 1(a). In the transfer characteristics of the OECTs (displayed in Fig. 1(b)), we observed an averaged transconductance of $1 \mu \mathrm{S}$ for the 40 measured OECTs: these wide spreads in device performances gives us the opportunity to probe the capability for our addressing circuits for various electrical properties of the OECT.

\section{OECT SENSOR CIRCUITS}

Here, the electronic setups are implemented to measure signals from a single OECT by using an analog measurement device (e.g Oscilloscope). The working mechanism of these electronic 
circuits relies on converting the drain current or the resistance of the OECT into voltage, signal amplification, and filtering.

- One setup considers OECTs as a current source sensors,

$$
V_{\text {out }}=\left(\frac{1}{2}-\frac{R_{\text {OECT }}+\Delta R}{R+\left(R_{O E C T}+\Delta R\right)}\right) V_{S}
$$

bridge circuit.

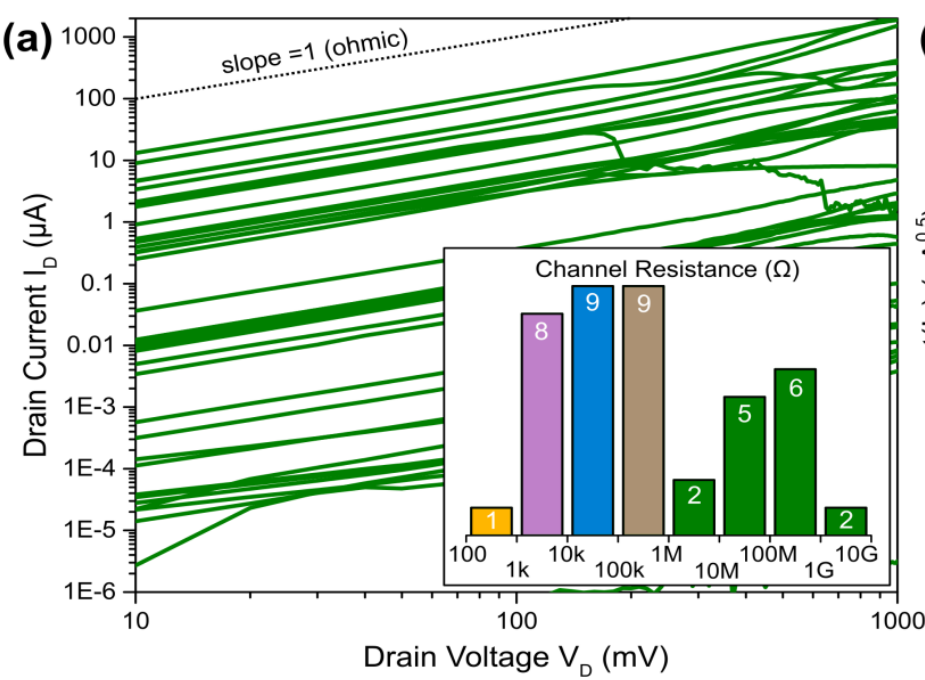

variation $(\triangle \mathrm{R})$ in the resistance of the OECT $\left(R_{O E C T}\right)$ that will induce a change in the output signal of the Wheatstone bridge described by (1).
If the load resistances are calibrated manually via

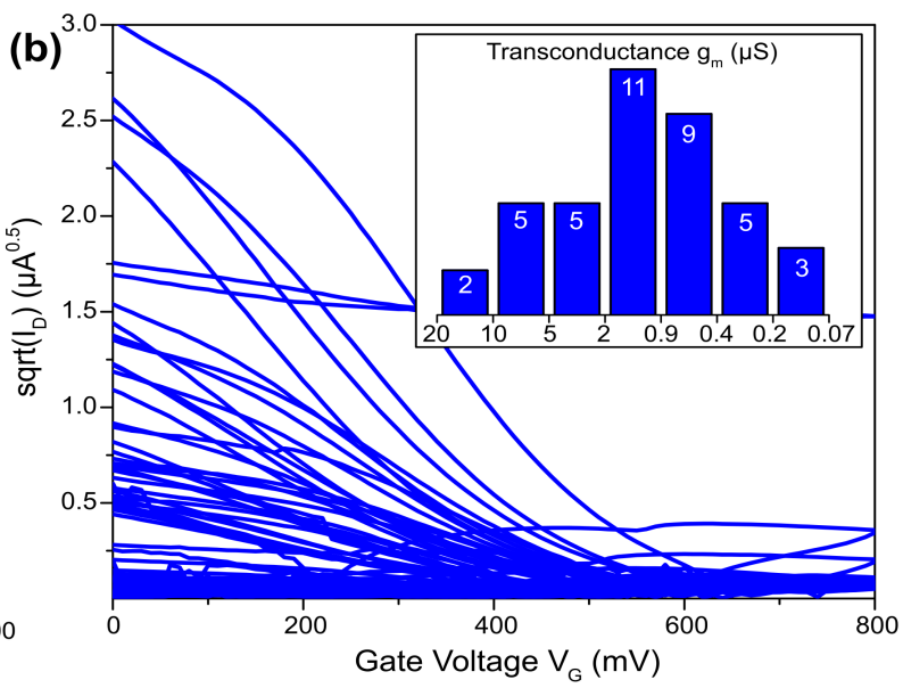

Fig. 1 Characteristics of the OECTs used to assess the circuits: (a) Output characteristics of a population displaying an Ohmic behavior in the applied voltage range (inset: histogram of their channel resistances). (b) Transfer characteristics of the same population (inset: histogram of their transconductance)

\section{A. OECT with a Transimpedance Configuration}

The working mechanism of this recording circuitry is shown in Fig. 2. Any electrical activity, coming from the electrogenic cells sensed by OECT as current modulations, is converted to voltage changes through the I/V convertor with a feedback resistor of $10 \mathrm{k} \Omega$ at the first stage. To remove the offset signal (at constant gate voltage) and the noise of the low frequency biochemical signals, the resulted voltage is filtered by a $3^{\text {rd }}$-order Butterworth high-pass filter with cutoff frequency $F_{1}=1 \mathrm{~Hz}$ (with $-60 \mathrm{~dB} /$ decade of attenuation below $\mathrm{F}_{1}$ ). The signal is then amplified by $50 \mathrm{X}$ at the third stage and then it is filtered by $3^{\text {rd }}$ order Butterworth low-pass filter with cutoff frequency $F_{h}=10 \mathrm{kHz}$ (with $-60 \mathrm{~dB} /$ decade of attenuation beyond $F_{h}$ ) to limit the noise bandwidth (Fig. 2).

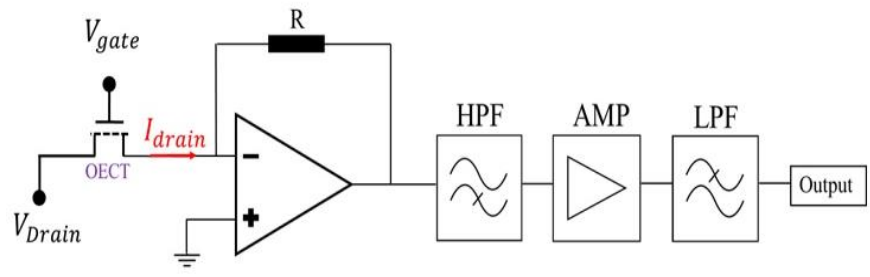

Fig. 2 Schematic of the OECT with the I-V converter circuit

\section{B. OECT with a Wheatstone Bridge Configuration}

Wheatstone bridge circuits are commonly used for differential resistance measurements. Due to its observed ohmic behavior, OECTs can be considered as resistive sensors implementable in a Wheatstone bridge with other load resistors $(\mathrm{R})$ as shown in Fig. 3. Any electrical activity in the electrolyte coming from the electrogenic cells sensed by the OECT will cause small

This work was funded by the European Commission under the ERC-2017-COG program, IONOS project GA773228. potentiometers such that they equal the one of the OECT $\left(R_{O E C T}\right)$ at rest, the output signal can be described by (2).

$$
V_{\text {out }}=\left(\frac{\Delta R}{R+\frac{\Delta R}{2}}\right) \frac{V_{C C}}{4}
$$

Where $\mathrm{V}_{\mathrm{CC}}$ is the low voltage supply $(100 \mathrm{mV})$. Therefore, the electrical activities of the cells will modulate the output voltage of the Wheatstone bridge that can be monitored later.

The resulted output voltage of the Wheatstone bridge is then amplified by a $100 \mathrm{X}$ differential amplifier. The amplified signal is then passed through high- and low-pass filters separated by a buffer. The filters configurations are implemented with the same type and design than the ones previously implemented in the Transimpedance configuration.

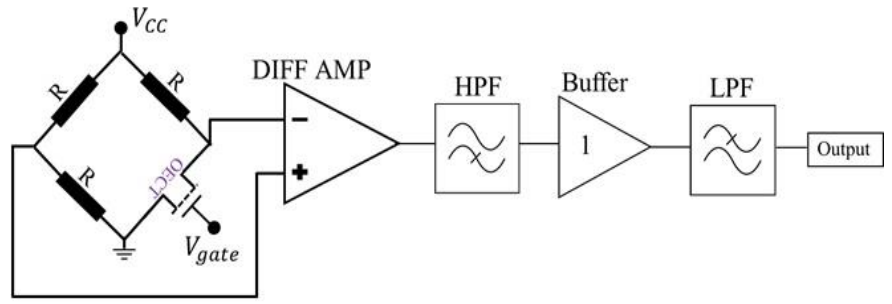

Fig. 3.Schematic of the OECT with the Wheatstone bridge configuration

\section{EXPERIMENTS AND RESULTS}

For each circuit configuration, a PCB circuit has been fabricated. The 42-OECT chip was interfaced with the PCB by wire bonding on a CLCC (Ceramic Leadless Chip Carrier) inserted in a PLCC (Plastic Leaded Chip Carrier) socket on the board (Fig. 4). In the Transimpedance configuration PCB, the 
OECTs drain-source channels are biased at the same time by a $100 \mathrm{mV}$ biasing voltage and each OECT output can be selected to connect the input of the circuit by a jumping wire. In the Wheatstone bridge configuration PCB, the source and the drain of each OECT can be selected and connected to the Wheatstone bridge circuit by jumping wires where each OECT will be biased from the alimentation input of the Wheatstone bridge (Fig. 4).

Despite no particular shielding, both electronic circuits achieved a noise level (root mean square) in the range of 10$20 \mathrm{mV}$ which was low enough to monitor clearly the electrical spikes at the output of these circuits as shown in Fig. 5.

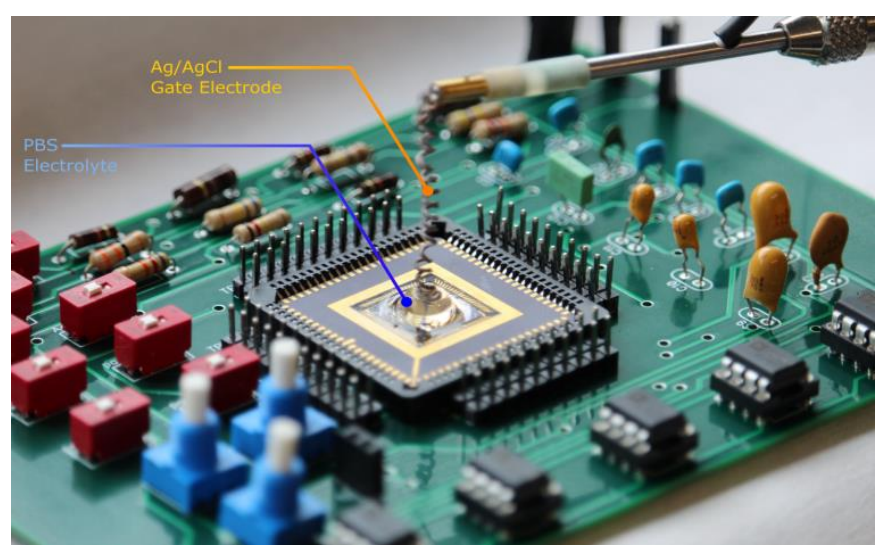

Fig. 4. Picture of the Wheatstone bridge configuration circuit with the $\mathrm{Ag} / \mathrm{AgCl}$ gate electrode dipped in the PBS electrolyte.

The experiments were done for investigating the response of the OECT output to transient signals (e.g. voltage pulses applied to the gate) in Phosphate-Buffered Saline (PBS). The fix drain potential (and the Wheatstone bridge circuit input bias for the Wheatstone bridge configuration) was set to $100 \mathrm{mV}$ and the output of each circuit was measured continuously at a pulsed gate voltage $\mathrm{V}_{\text {gate }}=100 \mathrm{mV}$ over a $0.5 \mathrm{~s}$ ( $2 \mathrm{~Hz}$ frequency) period with $1 \mathrm{~ms}$ pulse width. These experiments were tested on four different OECTs, from four different channel resistivity values as they were categorized in the histogram in Fig. 1a. As shown in the graphs in Fig. 5. Both circuits successfully recorded the simulated gate input signal, with the offset DC output signal being removed in both cases. Comparing both circuits by referring to Fig. $5 \mathrm{~b}$ and Fig. $5 c$, the value of the output of the I-V converter circuit is directly related to the resistivity of the OECT channel. For lower resistivity values, we got higher drain currents and thus higher output voltage. However, for the Wheatstone bridge circuit, in addition to the resistivity of the OECT, the output voltage depends also on the values of the fixed load resistances (as shown in (2)). Hence, Wheatstone bridge circuit is still sensitive for OECTs with high resistance values while on the other hand, the time of the charge and discharge is noticeably slower than the I-V converter output response.

\section{CONCLUSION}

This paper reports the implementation of two electronic circuits for addressing OECT for neuromorphic sensing applications. Electrical characterizations of the OECTs also been carried out to show the several electrical properties of the devices before testing them with the circuits. These circuits showed successful response after their tested on a simulated signal of the cells promising that it will provide effective results for real biological measurements for electrogenic cells.

\section{Acknowledgements}

We acknowledge financial supports from the EU: ERC-2017COG project IONOS (\# GA 773228). We thank the French National Nanofabrication Network RENATECH for financial support of the IEMN clean-room. We thank also the IEMN cleanroom staff of their advices and support 

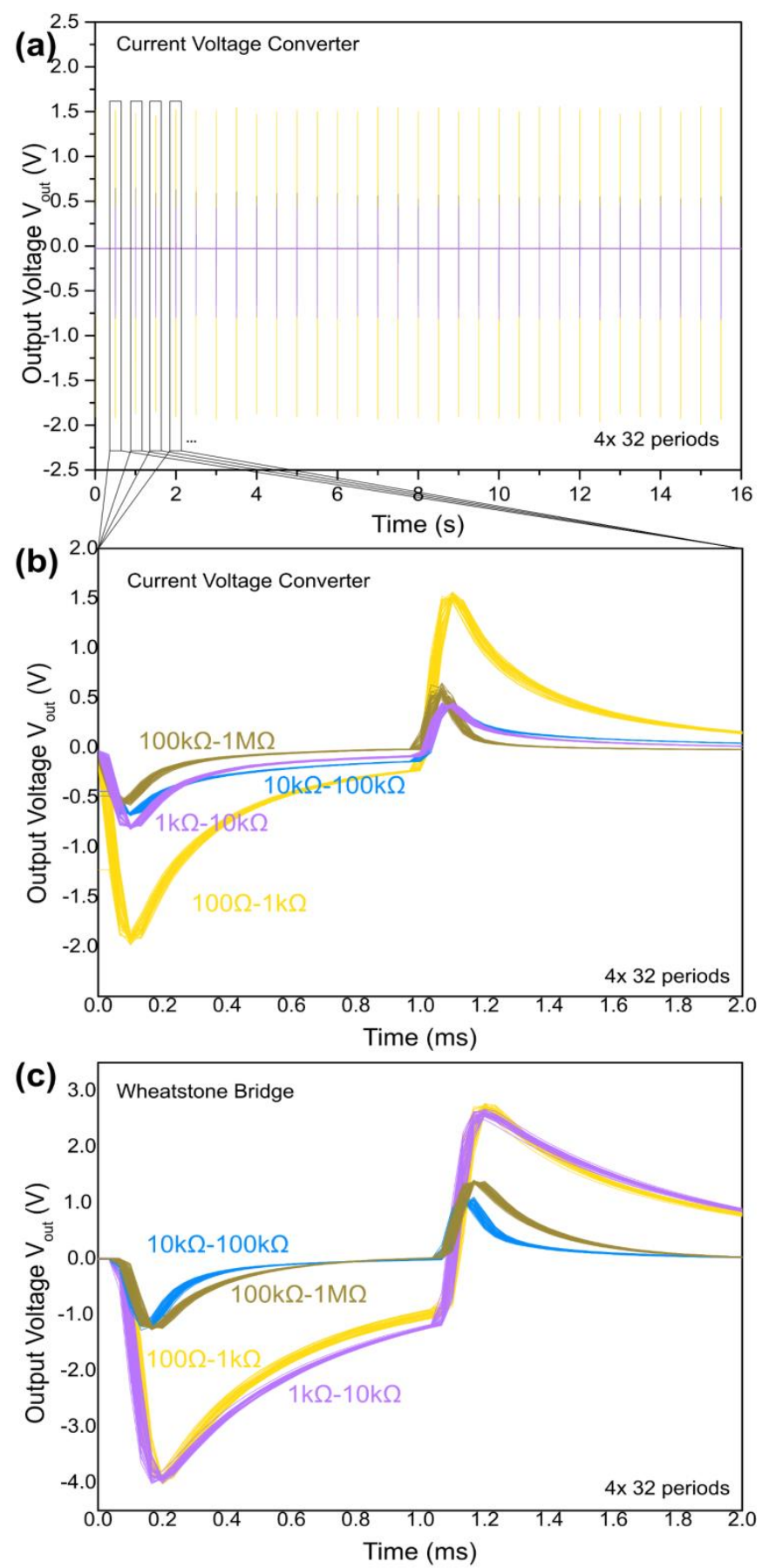

Fig. 5. Response of the output voltage on the simulated signals of electrogenic cells: (a) of the I-V converter circuit, (b) zoomed shot of one spike at the output of the I-V converter circuit, (c) zoomed shot of one spike at the output Wheatstone bridge circuits.

\section{REFERENCES}

[1] D. Khodagholy et al., « High transconductance organic electrochemical transistors », Nat. Commun., vol. 4, p. 2133, july 2013.

[2] S. V. Rajaraman, S. Choi, M. A. McClain, J. de R. Ross, M. C. LaPlaca, et M. G. Allen, « Metal-TransferMicromolded Three-Dimensional Microelectrode Arrays for in-vitro Brain-Slice Recordings », $J$.

Microelectromechanical Syst., vol. 20, p. 396-409, 2011.

[3] J. P. Spatz et B. Geiger, « Molecular engineering of cellular environments: cell adhesion to nano-digital surfaces », Methods Cell Biol., vol. 83, p. 89-111, 2007.

[4] D. Khodagholy et al., «Organic electrochemical transistor incorporating an ionogel as a solid state electrolyte for lactate sensing », J. Mater. Chem., vol. 22,

$$
\mathrm{n}^{\mathrm{o}} 10 \text {, p. 4440-4443, feb. } 2012 .
$$

[5] C. Liao, M. Zhang, L. Niu, Z. Zheng, et F. Yan, « Organic electrochemical transistors with graphenemodified gate electrodes for highly sensitive and selective dopamine sensors », J. Mater. Chem. B, vol. 2, n 2, p.

191-200, dec. 2013.

[6] «Organic Electrochemical Transistor Arrays for In Vitro Electrophysiology Monitoring of 2D and 3D Cardiac

Tissues ».

[7] C. Liao, M. Zhang, M. Y. Yao, T. Hua, L. Li, et F. Yan, «Flexible Organic Electronics in Biology: Materials and Devices », Adv. Mater. Deerfield Beach Fla, vol. 27, $\mathrm{n}^{\circ}$

$$
\text { 46, p. 7493-7527, dec. } 2015 .
$$

[8] M. E. Spira et A. Hai, « Multi-electrode array technologies for neuroscience and cardiology », Nat.

Nanotechnol., vol. 8, n 2, p. 83-94, feb. 2013.

[9] S. Pecqueur, D. Guérin, D. Vuillaume, F. Alibart, Cation Discrimination in Organic Electrochemical Transistors by Dual Frequency Sensing, Org. Electron. 57 (2018), 232-238. 